\title{
A phase II, open-label study of gefitinib (IRESSA) in patients with locally advanced, metastatic, or relapsed renal-cell carcinoma
}

Received: 14 April 2005/ Accepted: 22 June 2005 / Published online: 29 July 2005

(C) Springer-Verlag 2005

\begin{abstract}
Epidermal growth factor receptor (EGFR) expression has been associated with clinical outcome in some studies of renal-cell carcinoma (RCC). We investigated the efficacy and safety of gefitinib (IRESSA), an EGFR tyrosine kinase inhibitor, in RCC patients. This phase II trial recruited 28 patients with advanced, metastatic, or relapsed RCC. Patients received oral gefitinib $500 \mathrm{mg} /$ day. Objective responses (ORs) were assessed every 2 months according to RECIST. Baseline tumor biopsies were analyzed immunohistochemically for EGFR expression. At trial closure (March 2003), no ORs were seen but 14 patients $(53.8 \%)$ had stable disease. At extended analysis (August 2004), median time to progression was 110 days $(95 \%$ confidence interval [CI]: 55, 117); median overall survival was 303 days $(95 \%$ CI 180,444$)$. Gefitinib was generally well tolerated. Skin rash and diarrhea were the most common drug-related adverse events (AEs) [54 and 39\% of patients, respectively] and the most common drug-re-
\end{abstract}

M. Jermann $(\bowtie) \cdot R$. A Stahel

Clinic and Policlinic of Oncology, University Hospital, Zurich, Switzerland

M. Salzberg $\cdot$ M. Pless

Department of Medicine and Oncology, University Hospital, Basel, Switzerland

T. Cerny $\cdot$ M. Joerger $\cdot$ S. Gillessen

Department of Medicine, Oncology and Hematology, Cantonal Hospital, St Gallen, Switzerland

R. Morant

ZeTuP, St Gallen, Switzerland

F. Egli

Medical Clinic, Cantonal Hospital, Chur, Switzerland

K. Rhyner

Medical Clinic, Cantonal Hospital, Glarus, Switzerland

J. A Bauer

Oncology Centre, CHUV, Lausanne, Switzerland

M. Jermann

Department of Oncology, Medical University Clinic, 4101, Bruderholz, Switzerland lated grade $3 / 4 \mathrm{AEs}$ (both $11 \%$ ). The majority of tumor biopsies $(91 \%)$ had $\geq 70 \%$ of tumor cells expressing membrane EGFR. Despite the lack of ORs in this study, disease control was observed in $53.8 \%$ of patients. Gefitinib was generally well tolerated and no unexpected drug-related AEs were observed.

Keywords EGFR - TKI - Gefitinib - IRESSA · Renal-cell carcinoma

\section{Introduction}

In 2002, renal-cell carcinoma (RCC) accounted for 4\% of adult malignancies globally, with $>200,000$ cases diagnosed and $\sim 100,000$ deaths reported [7]. For patients with metastatic or recurrent RCC, treatment options are limited, prognosis is poor, and median survival is $<1$ year [16]. Cytotoxic chemotherapy regimens are relatively ineffective and most chemotherapy trials demonstrate no clear survival benefit [9]. While those patients who do respond to chemotherapy often survive longer, only few patients show tumor regression (e.g., 5-9\% of patients experience objective responses (ORs) with agents such as vinblastine and 5-fluorouracil [5-FU]).

Various immunologic agents have been evaluated, including interleukin 2 (IL-2) (2-18 million units $\left.[\mathrm{MU}] / \mathrm{m}^{2}\right)$ and interferon alpha $(\mathrm{IFN}-\alpha)(3-15 \mathrm{MU} /$ day) [24]. Both drugs have a similar OR rate (complete response [CR] plus partial response [PR]) of 10 $20 \%$ in appropriately selected patients (non-bulky pulmonary and/or soft tissue metastases with performance status $0-1$ and no weight loss), with an additional $20-30 \%$ of patients experiencing stable disease (SD) $[2,10,19,24]$. However, only patients treated with IL-2 experience durable ( $>5$ years) CR $(5 \%$ of patients); those treated with IFN- $\alpha$ rarely have complete or durable responses [24].

Combining IL-2 and IFN- $\alpha$ can increase efficacy end points slightly, with OR and SD observed in $\leq 40 \%$ of 
patients [2, 10, 19]. The clinical benefit gained using immunotherapy may be offset by its toxicity, which can be substantial; grade 3/4 adverse events (AEs) consist of fatigue, weight loss, fever, myalgia, hypotension, depression, and hematologic toxicities (e.g., neutropenia, thrombocytopenia, and anemia) [10, 19].

While combination regimens including chemotherapy agents (e.g., 5-FU or vinblastine) do not improve OR and SD, they may improve survival end points $[3,18$, 21]. In a more recent outpatient regimen, a significant prolongation in progression-free survival (PFS) was observed for patients with advanced RCC treated with IFN- $\alpha+$ IL2 +5 -FU + cis-retinoic acid (7 months) compared with IFN- $\alpha+$ vinblastine (5 months). However, there was no improvement in PFS with IFN- $\alpha+$ IL2 +5 FU compared with IFN- $\alpha+$ IL $2+5-\mathrm{FU}+$ cis-retinoic acid (6 months) [3]. Similar trends were also observed for OS. The percentage of patients who discontinued treatment due to toxicity was $4 \%$ in the IFN- $\alpha+$ IL $2+5$ FU arm, $6 \%$ in the IFN- $\alpha+$ IL $2+5-\mathrm{FU}+$ cis-retinoic acid arm, and $8 \%$ in the IFN- $\alpha+$ vinblastine arm [3]. Clearly there is a need for new, effective, and well-tolerated therapies for the management of RCC.

Approximately $70-90 \%$ of RCC cases express the epidermal growth factor receptor (EGFR) [1,23], which is known to be involved in key tumorigenic processes such as proliferation, invasion, survival, and angiogenesis [12, 14, 25, 27, 29]. EGFR expression has been associated with clinical outcome in some studies of RCC. For example, reduced survival and an increased risk of metastatic disease have been observed in patients whose tumors express EGFR [14, 25, 29], while less regional lymph node involvement, venous invasion, and distant metastases were observed in patients whose tumors did not express EGFR [29]. These data suggest that EGFR inhibition may be a valid treatment option in RCC.

Inhibition of EGFR signaling has been shown to be effective in the non-small-cell lung cancer setting. Phase II trials showed that $11.8-18.4 \%$ of pretreated patients with locally advanced or metastatic non-small-cell lung cancer experienced ORs following treatment with $250 \mathrm{mg}$ /day gefitinib (IRESSA), the first EGFR tyrosine kinase inhibitor to be approved for the treatment of cancer [6]. The majority of drug-related AEs were grade $1 / 2$ mild to moderate diarrhea and skin rash, with few patients experiencing grade $3 / 4$ AEs $[6,11]$.

In the absence of efficacy data in RCC at the time that this phase II trial was designed, a dose of $500 \mathrm{mg} /$ day was used to investigate the efficacy and tolerability of gefitinib in patients with advanced, metastatic, or relapsed RCC.

\section{Methods}

Trial design and patients

This was an open-label, multicenter, non-comparative, phase II trial. The primary objective was to determine the
OR rate (CRs and PRs) of $500 \mathrm{mg} /$ day gefitinib in patients with advanced, metastatic, or relapsed RCC. Secondary objectives included estimating the disease control rate, time to progression (TTP) and OS, and duration of response, as well as further evaluation of the safety of gefitinib at a dose of $500 \mathrm{mg} / \mathrm{day}$. An exploratory objective was to investigate any association between baseline tumor EGFR expression and response to gefitinib.

Inclusion criteria consisted of: histologically confirmed, locally advanced, relapsed or metastatic RCC that was refractory to, or intolerant of, approved therapies; age $\geq 18$ years; World Health Organization performance status 0-2 or Karnofsky score $>70 \%$; life expectancy of $>12$ weeks; measurable disease according to the modified response evaluation criteria in solid tumors (RECIST). Patients with local tumor resection were eligible only if local relapse or distant progression had been radiologically documented.

Any of the following was regarded as a criterion for exclusion: nephrectomy within 4 weeks of the trial starting; other co-existing malignancies or malignancies diagnosed within the previous 5 years (with the exception of basal-cell carcinoma or cervical cancer in situ); any unresolved chronic toxicity greater than National Cancer Institute Common Toxicity Criteria (NCI-CTC) grade 2 from previous anticancer therapy; incomplete healing from previous oncologic or other surgery; absolute neutrophil count $<1.0 \times 10^{9} / \mathrm{L}$ or platelets $<100 \times 10^{9} / \mathrm{L}$, serum bilirubin $>1.25 \times$ upper limit of reference range (ULRR); any evidence of severe or uncontrolled systemic disease; serum creatinine $>160 \mu \mathrm{mol} / \mathrm{L}$; alanine aminotransferase or aspartate aminotransferase $>2.5 \times$ ULRR if no liver metastases were present or $>5 \times$ ULRR in the presence of liver metastases; evidence of brain metastases; chemotherapy or cytokine therapy in the 4 weeks prior to enrollment; radiotherapy within 1 week of enrollment, concomitant use of CYP3A4 inhibitors (e.g., phenytoin, carbamazepine, barbiturates, rifampicin, or St John's Wort); pregnancy or breastfeeding (women of childbearing potential); any psychiatric disorder that might restrict patient compliance; patients known to be, or at risk of being, HIV + .

The trial was conducted in accordance with local and international regulatory and ethical guidelines, and all patients gave written, informed consent.

\section{Treatment}

Patients received oral gefitinib $500 \mathrm{mg} /$ day (two $250 \mathrm{mg}$ tablets) in the morning as a continuous treatment, at approximately the same time each day. In the event of toxicity, gefitinib treatment could be interrupted for $\leq 14$ days, with repeated drug interruptions allowed, as necessary. If toxicity recurred once gefitinib therapy recommenced and dose interruption was not considered sufficient to manage the toxicity, the dose could be reduced to $250 \mathrm{mg} / \mathrm{day}$. 
Patients could be withdrawn from the study at any time at the discretion of the investigating physician. Discontinuation criteria included: withdrawal of informed consent; objective disease progression; patient lost to follow-up; initiation of additional antineoplastic therapies; AEs; protocol non-compliance; death.

\section{Assessments}

\section{Efficacy}

Objective responses (CRs and PRs) were evaluated using RECIST. X-rays and computed tomography (CT) scans were carried out within 3 weeks of study entry. Tumors were assessed by CT scan every 8 weeks during the first 6 months of therapy; thereafter, X-rays and CT scans were carried out every 12 weeks. Duration of response was to be calculated as the number of days from the date of first documented response to the earlier of death (from any cause) or progression, and the last on-study tumor assessment. The disease control rate was defined as those patients with ORs plus those with SD confirmed and sustained for $\geq 4$ weeks.

TTP was calculated as the number of days from the day of first treatment to the earlier of death (from any cause) or progression, and the last on-study tumor assessment. OS was assessed as the number of days from the day of first treatment to the earlier of death (from any cause), and the last date of patient contact.

\section{Tolerability}

AEs were recorded throughout the study using NCICTC version 2.0 and causality was assigned by the investigators. Routine hematology, biochemistry, and physical examinations were carried out within 7 days before the start of treatment, on days 1, 15, and 29 of treatment, and every 28 days thereafter. Urinalysis was performed as necessary.

\section{Tumor EGFR expression}

Tumor EGFR expression levels could be determined in 23 patients from tumor biopsies taken within 21 days of starting gefitinib. Immunohistologic evaluation of tumor EGFR expression was carried out at an AstraZenecadesignated laboratory using EGFR pharmDx (DakoCytomation).

\section{Statistical analysis}

Fleming's method was used to calculate the number of patients required, with 27 patients being sufficient to give an $80 \%$ probability of rejecting a baseline response rate of $5 \%$ with an exact one-sided significance test when the true response is at the clinically relevant rate of $20 \%$.
The population for all analyses was the intent-to-treat (ITT) population. The null hypothesis that the OR rate was $5 \%$ was to be tested against the alternative hypothesis that the response rate was $>5 \%$. If there were 27 patients in the ITT population and $\geq 4$ of these patients were responders, the null hypothesis was to be rejected. If the number of patients in the ITT population differed from 27, the null hypothesis was to be rejected in favor of the alternative hypothesis if the $90 \%$ confidence interval (CI) did not include 5\%.

The summary statistics for continuous variables were mean, standard deviation, median, quartiles, minimum, and maximum. Discrete variables were summarized by count and proportion. OR and disease control rates were summarized by proportions, together with a $95 \%$ CI (a 90\% CI was also calculated for the OR rate). Durations (TTP, OS, and response) were summarized by Kaplan-Meier methods.

An exact logistic regression model would calculate the regression coefficient of logs-odd tumor response against EGFR expression, together with a 95\% CI.

\section{Results}

\section{Patient characteristics}

Twenty-eight caucasian patients (19 men, 9 women) were enrolled into this study from March to September 2002. All patients had metastatic disease. The baseline patient characteristics are shown in Table 1. As a per-protocol analysis was not performed, protocol deviations were not assessed. Twenty-four patients met all inclusion and exclusion criteria. Only one patient had a cytologic analysis of tumor tissue. Three patients failed the exclusion criterion of having serum creatinine $\leq 160 \mu \mathrm{mol} / \mathrm{L}$; however, as the level for all three was $<190 \mu \mathrm{mol} / \mathrm{L}$ they were all included in the study.

\section{Treatment}

All patients received at least one dose of gefitinib. Median time on trial was 64.5 days (range: 8-354), with the median duration of treatment being 63 days (range: 8-345).

\section{Efficacy}

Twenty-six of 28 patients were evaluable for efficacy. Of the two patients who were not evaluable for efficacy, one patient died after 42 days of treatment (before the first radiologic assessment) and the other withdrew informed consent after 13 days due to minor toxicities. Per the trial protocol, efficacy data were analyzed at trial closure (March 2003); however, because median survival had 
not been reached at this time, a second analysis was performed in August 2004.

Although none of the patients in this study experienced an OR, disease was controlled in 14 of the 26 evaluable patients $(53.8 \%$; 95\% CI: 33.4, 73.4) at their first radiological assessment at 8 weeks.

At trial closure, 16 patients were alive, and 12 had died (in whom PD was not documented in two patients). Median TTP was 99 days $(95 \%$ CI: 55-114) and 6 months after starting treatment, six patients $(21.4 \%$; $96 \%$ CI: $6.2-36.6 \%$ ) were alive and progression free. As 16 patients $(57.1 \%)$ were alive at trial closure, the median OS could not be estimated.

A more recent efficacy analysis in August 2004 showed that 24 patients had progressed and 22 had died. Five patients had experienced prolonged SD for $\geq 1$ year (range: 12-26+ months), including one patient with clear-cell RCC with multiple lung metastases and distant lymph node involvement who is still receiving gefitinib (duration: $26+$ months). Two patients with SD withdrew informed consent after 5.7 and 15 months, respectively; the former had documented ongoing SD 18.1 months after discontinuation of gefitinib treatment, and the latter had disease progression 9 months after discontinuation of gefitinib therapy. Median TTP was 110 days $(95 \%$ CI: 55,117$)$ and median OS was 303 days (95\% CI: 180, 444) (Fig. 1a, b).

\section{Tolerability}

All 28 patients were evaluable for safety and the majority of patients $(n=26,93 \%)$ experienced AEs. Drug-related AEs occurred in 25 patients $(89 \%)$, the most frequent being grade $1 / 2$ skin rash $(54 \%)$ and diarrhea $(39 \%)$ (Table 2). Few drug-related grade $3 / 4$ AEs occurred, with the most common being skin rash (11\% of patients) and diarrhea $(11 \%)$. One of these patients experienced grade 4 diarrhea (leading to dehydration) and grade 4 renal failure. Another patient experienced grade 3 febrile neutropenia (he had not received prior chemotherapy but had received spinal and pelvic radiotherapy) and grade 4 deterioration in physical health; this patient later died from a grade 4 cerebrovascular accident that was not considered to be drug related.

Drug-related AEs led to dose interruptions and reductions in seven $(25 \%)$ and six patients $(21 \%)$, respectively. Two patients $(7 \%)$ discontinued treatment because of AEs; the patient mentioned above with drugrelated grade 4 deterioration in general condition, and one patient who had drug-related grade 3 skin rash, which resolved after treatment discontinuation. There were no drug-related deaths.

\section{EGFR expression and response}

Twenty-three tumor biopsies were available for EGFR analysis, with the majority of samples $(91 \%)$ having $\geq 70 \%$ of tumor cells expressing membrane staining for EGFR (highest intensity of $2+$ or $3+$ ). As no ORs were observed, the exploratory analysis could not be performed. Three out of the five patients with prolonged SD had tumor biopsies available for analysis (two patients did not provide a biopsy sample); one patient had strong EGFR expression $(95 \%$ membrane staining with $50 \%+++$ ) and two patients had weak membrane staining. Information on EGFR expression for the patient with ongoing SD is not available.

\section{Discussion}

This study has shown that the administration of $500 \mathrm{mg} /$ day gefitinib to patients with advanced, metastatic, or relapsed RCC did not result in ORs. However, disease control was observed in $53.8 \%$ of patients at 8 weeks, and the median TTP and OS were 3.6 and 10.3 months, respectively. Treatment with $500 \mathrm{mg} /$ day gefitinib was generally well tolerated and no unexpected drug-related AEs were observed.

Our results are comparable to other reported phase II trials of $500 \mathrm{mg} /$ day gefitinib in patients with advanced RCC. In a study by Drucker et al. [5] involving 16 evaluable patients with advanced RCC, there were no ORs but disease control was seen in nine patients $(56 \%)$, three of whom experienced SD for $>4$ months. Similarly, no ORs were reported in an abstract with preliminary data by Dawson et al. [4] of 21 evaluable patients with advanced RCC. One patient had a minor response $(27 \%$ reduction in tumor size) and eight patients $(38 \%)$ had SD. Median PFS and OS were 2.7 and 8.3 months, respectively [4]. This lack of ORs in patients with metastatic RCC has also been observed in a phase II trial of the EGFR monoclonal antibody cetuximab (ABX-EGF) [15], where TTP was not prolonged with cetuximab compared with historical IFN- $\alpha$ data. However, a recent report showed that cetuximab induced major responses in three out of 88 patients with metastatic RCC and minor responses in two patients [23]. Median PFS was 100 days, which is comparable to our data, and the authors noted a trend that the severity of rash may relate to PFS [23].

Overall, these studies suggest that EGFR-targeted monotherapy results in a minimal OR rate in patients with advanced or metastatic RCC, even though correlations between EGFR expression and clinical outcome have been reported $[1,12,14,29]$. These clinical findings for gefitinib in the RCC setting are disappointing, considering that preclinical data have shown that gefitinib inhibits the proliferation of RCC cell lines (SKRC-49) and significantly inhibits the growth of RCC xenografts (SKRC-49) compared with placebo $(P<0.01)$ [1]. Moreover, the combination of gefitinib and paclitaxel significantly increased SKRC-49 cell apoptosis in vitro and tumor growth in vivo compared with gefitinib alone [26]. While no data are available as yet, analysis of renal biopsies for EGFR mutations 
Fig. 1 a Time to progression and $\mathbf{b}$ overall survival in patients with renal-cell carcinoma treated with $500 \mathrm{mg} /$ day gefitinib
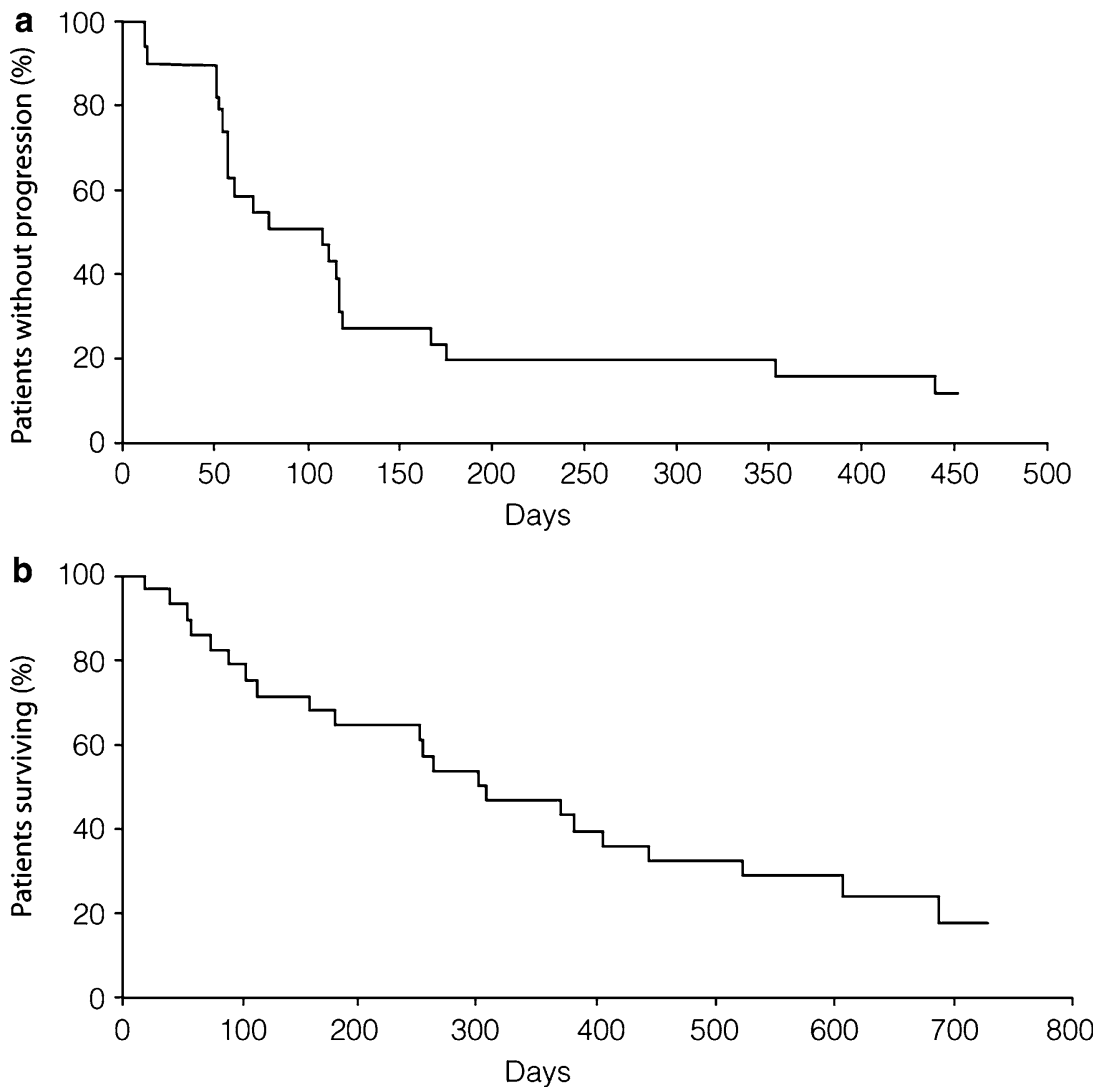

Table 1 Patient characteristics at baseline $(n=28)$

\begin{tabular}{ll}
\hline Median age (range), years & $63(37-75)$ \\
\hline Gender, $n(\%)$ & \\
Male & $19(68)$ \\
Female & $9(32)$ \\
World health organization performance status, $n(\%)$ & \\
0 & $12(43)$ \\
1 & $13(46)$ \\
2 & $3(11)$ \\
Prior therapy, ${ }^{\text {a }} n(\%)$ & $13(46)$ \\
None & $6(21)$ \\
Immunotherapy & $4(14)$ \\
Radiotherapy & $1(4)$ \\
Chemotherapy & $4(14)$ \\
Immunotherapy and chemotherapy & \\
No. metastatic sites, ${ }^{b} n(\%)$ & $2(7)$ \\
1 & $7(25)$ \\
2 & $8(29)$ \\
3 & $11(39)$ \\
$\geq 4$ & $18(64)$ \\
Sites of metastases, ${ }^{b} n(\%)$ & $12(43)$ \\
Lung & $8(29)$ \\
Lymph nodes & $7(25)$ \\
Liver & $5(18)$ \\
Mediastinum & $5(18)$ \\
Renal & $4(14)$ \\
Adrenal & $3(11)$ \\
Other &
\end{tabular}

${ }^{\text {a }}$ Patients may have received $>1$ type of prior therapy

${ }^{\mathrm{b}}$ Patients may have $>1$ metastatic site (recently observed in patients with non-small-cell lung cancer who had striking responses to gefitinib $[13,20])$ may shed light on the minimal OR rate and prolonged SD observed in patients with RCC receiving EGFRtargeted therapies.

More recently, several phase II trials have shown that targeted inhibition of more than one signaling pathway associated with $\mathrm{RCC}$ is effective in patients with metastatic disease $[8,17,22]$. Twenty-five out of 65 evaluable patients $(38 \%)$ responded to BAY $43-$ 9006, a novel signal transduction inhibitor that targets the platelet-derived growth factor receptor (PDGFR) and vascular endothelial growth factor receptor (VEGFR) pathways [22], and Motzer et al. [17] reported ORs in 15 out of 63 patients $(24 \%)$ who were treated with SU11248, which inhibits the PDGFR, VEGFR, KIT, and FLT-3 signaling pathways. ORs were also observed in 10 out of 40 evaluable patients $(25 \%)$ following blockade of VEGFR and EGFR using bevacizumab (an anti-VEGF monoclonal antibody) and erlotinib (an EGFR tyrosine kinase inhibitor) [8]. Blockade of VEGFR alone has also shown efficacy in metastatic RCC, with a randomized phase II trial of bevacizumab and placebo in patients with metastatic RCC showing significantly prolonged TTP in favor of bevacizumab (4.8 vs. 2.5 months) [28]. Whether gefitinib in combination with other targeted therapies or with cytotoxic chemotherapy agents is active in patients with RCC remains to be determined. 
Table 2 Drug-related adverse events occurring in $\geq 10 \%$ of patients and all drug-related grade $3 / 4$ adverse events

\begin{tabular}{llll}
\hline Adverse event & Grade $1 / 2, n(\%)$ & Grade $3, n(\%)$ & Grade $4, n(\%)$ \\
\hline Skin rash $^{\mathrm{a}}$ & $15(53.6)$ & $3(10.7)$ & $0(0.0)$ \\
Diarrhea & $11(39.3)$ & $2(7.1)$ & $1(3.6)^{\mathrm{b}}$ \\
Dry skin & $8(28.6)$ & $0(0.0)$ & $0(0.0)$ \\
Pruritus & $3(10.7)$ & $1(3.6)$ & $0(0.0)$ \\
Acne & $3(10.7)$ & $0(0.0)$ & $0(0.0)$ \\
Dry eye & $3(10.7)$ & $0(0.0)$ & $0(0.0)$ \\
Vomiting & $3(10.7)$ & $0(0.0)$ & $0(0.0)$ \\
Fatigue & $2(7.1)$ & $1(3.6)$ & $0.0)$ \\
Decreased appetite & $1(3.6)$ & $1(3.6)$ & $0(0.0)$ \\
Conjunctivitis & $1(3.6)$ & $1(3.6)$ & $0.0)$ \\
Febrile neutropenia & $0(0.0)$ & $1(3.6)^{\mathrm{c}}$ & $0.0)$ \\
Skin lesion & $0(0.0)$ & $0(0.0)$ & $1(3.0)$ \\
Deterioration in physical health & $0(0.0)$ & $0(0.0)$ & $1(3.6)^{\mathrm{b}}$ \\
Renal failure & $0(0.0)$ & & \\
\hline
\end{tabular}

${ }^{a}$ Includes papular and pustular rash, erythema (plantar erythema, palmar-plantar erythrodysesthesia syndrome), and exanthema

${ }^{\mathrm{b}}$ Occurred in the same patient

${ }^{c}$ Occurred in the same patient

\section{Conclusions}

In this study, gefitinib did not produce any ORs in patients with locally advanced, relapsed, or metastatic RCC. However, disease control was observed in 53.8\% of patients and median TTP and OS were 110 and 303 days, respectively. These findings are consistent with other monotherapy trials of gefitinib and other EGFR inhibitors. There were no unusual safety concerns, AEs being comparable to those previously reported. Despite the lack of ORs in this study, the level of disease control, along with preclinical and early clinical combination data, suggests that combination studies that include inhibition of the EGFR signaling pathways should be investigated further.

IRESSA is a trademark of the AstraZeneca group of companies

Acknowledgements This study was funded by AstraZeneca.

\section{References}

1. Asakuma J, Sumitomo M, Asano T et al (2004) Modulation of tumor growth and tumor induced angiogenesis after epidermal growth factor receptor inhibition by ZD1839 in renal cell carcinoma. J Urol 171:897-902

2. Atzpodien J, Kirchner H, Illiger HJ et al (2001) IL-2 in Combination with IFN-alpha and 5-FU versus tamoxifen in metastatic renal cell carcinoma: long-term results of a controlled randomized clinical trial. Br J Cancer 85:1130-1136

3. Atzpodien J, Kirchner H, Jonas U et al (2004) Interleukin-2and interferon alfa-2a-based immunochemotherapy in advanced renal cell carcinoma: a prospectively randomized trial of the German Cooperative Renal Carcinoma Chemoimmunotherapy Group (DGCIN). J Clin Oncol 22:1188-1194

4. Dawson NA, Guo C, Zak R et al (2004) A phase II trial of gefitinib (Iressa, ZD1839) in stage IV and recurrent renal cell carcinoma. Clin Cancer Res 10:7812-7819

5. Drucker B, Bacik J, Ginsberg M et al (2003) Phase II trial of ZD1839 (IRESSA) in patients with advanced renal cell carcinoma. Invest New Drug 21:341-345
6. Fukuoka M, Yano S, Giaccone G et al (2003) Multi-institutional randomized Phase II trial of gefitinib for previously treated patients with advanced non-small-cell lung cancer. J Clin Oncol 21:2237-2246

7. Globocan. Globocan 2002. Cancer incidence, mortality and prevalence worldwide. http://www-depdb.iarc.fr/globocan/ GLOBOframe.htm 2002.

8. Hainsworth JD, Sosman JA, Spigel DR et al (2004) Phase II trial of bevacizumab and erlotinib in patients with metastatic renal carcinoma (RCC). Proc Am Soc Clin Oncol 23:381 (Abstr 4502)

9. Hartmann JT, Bokemeyer C (1999) Chemotherapy for renal cell carcinoma. Anticancer Res 19:1541-1543

10. Jonasch E, Haluska FG (2001) Interferon in oncological practice: review of interferon biology, clinical applications, and toxicities. Oncologist 6:34-55

11. Kris MG, Natale RB, Herbst RS et al (2003) Efficacy of gefitinib, an inhibitor of the epidermal growth factor receptor tyrosine kinase, in symptomatic patients with non-small cell lung cancer. A Randomized Trial. JAMA 290:2149-2158

12. Lager DJ, Slagel DD, Palechek PL (1994) The expression of epidermal growth factor receptor and transforming growth factor alpha in renal cell carcinoma. Mod Pathol 7:544-548

13. Lynch TJ, Bell DW, Sordella R et al (2004) Activating mutations in the epidermal growth factor receptor underlying responsiveness of non-small-cell lung cancer to gefitinib. N Engl J Med 350:2129-2139

14. Moch H, Sauter G, Buchholz N et al (1997) Epidermal growth factor receptor expression is associated with rapid tumor cell proliferation in renal cell carcinoma. Hum Pathol 28:1255-1259

15. Motzer RJ, Amato R, Todd M et al (2003) Phase II trial of antiepidermal growth factor receptor antibody $\mathrm{C} 225$ in patients with advanced renal cell carcinoma. Invest New Drug 21:99-101

16. Motzer RJ, Bander NH, Nanus DM (1996) Renal-cell carcinoma. N Engl J Med 335: 865-875

17. Motzer RJ, Rini BI, Michaelson MD et al (2004) SU011248, a novel tyrosine kinase inhibitor, shows antitumor activity in second-line therapy for patients with metastatic renal cell carcinoma: Results of a phase 2 trial. Proc Am Soc Clin Oncol 23:381 (Abstr 4500)

18. Négrier S, Caty A, Lesimple T et al (2000) Treatment of patients with metastatic renal carcinoma with a combination of subcutaneous interleukin-2 and interferon alfa with or without fluorouracil. J Clin Oncol 18:4009-4015 
19. Negrier S, Escudier B, Lasset C et al (1998) Recombinant human interleukin-2, recombinant human interferon alfa-2a, or both in metastatic renal-cell carcinoma. Groupe Francais D'Immunotherapie. N Engl J Med 338:1272-1278

20. Paez JG, Jänne PA, Lee JC et al (2004) EGFR mutations in lung cancer: correlation with clinical response to gefitinib therapy. Science 304:1497-1500

21. Pyrhönen S, Salminen E, Ruutu M et al (1999) Prospective randomized trial of interferon alfa-2a plus vinblastine versus vinblastine alone in patients with advanced renal cell cancer. J Clin Oncol 17:2859-2867

22. Ratain MJ, Flaherty KT, Stadler WM et al (2004) Preliminary antitumor activity of BAY 43-9006 in metastatic renal cell carcinoma and other advanced refractory solid tumors in a phase II randomized discontinuation trial (RDT) Proc Am Soc Clin Oncol 23:381 (Abstr 4501)

23. Rowinsky EK, Schwartz GH, Gollob JA et al (2004) Safety, pharmacokinetics, and activity of ABX-EGF, a fully human anti-epidermal growth factor receptor monoclonal antibody in patients with metastatic renal cell cancer. J Clin Oncol 22:30033015
24. Sternberg CN (2003) Metastatic renal cell cancer treatments. Drugs Today (Barc) 39 (Suppl C):39-59

25. Stumm G, Eberwein S, Rostock-Wolf S et al (1996) Concomitant overexpression of the EGFR and ErbB-2 genes in renal cell carcinoma (RCC) is correlated with dedifferentiation and metastasis. Int J Cancer 69:17-22

26. Sumitomo M, Asano T, Asakuma J et al (2004) ZD1839 modulates paclitaxel response in renal cancer by blocking paclitaxel-induced activation of the epidermal growth factor receptor-extracellular signal-regulated kinase pathway. Clin Cancer Res 10:794-801

27. Woodburn JR (1999) The epidermal growth factor receptor and its inhibition in cancer therapy. Pharmacol Ther 82:241250

28. Yang JC, Haworth L, Sherry RM, et al (2003) A randomized trial of bevacizumab, an anti-vascular endothelial growth factor antibody, for metastatic renal cancer. N Engl J Med 349: 427-434

29. Yoshida K, Tosaka A, Takeuchi S et al (1994) Epidermal growth factor receptor content in human renal cell carcinomas. Cancer 73:1913-1918 